\title{
Metabolomic characterization of human hippocampus from drug-resistant epilepsy with mesial temporal seizure
}

\author{
Julien Detour $^{1,2}$ | Caroline Bund ${ }^{1,3}$ | Charles Behr ${ }^{4}$ | Hélène Cebula ${ }^{5}$ Ercument \\ A. Cicek ${ }^{6,7}$ | Maria-Paola Valenti-Hirsch ${ }^{4}$ | Béatrice Lannes ${ }^{8}$ | Benoît Lhermitte ${ }^{8}$ | \\ Astrid Nehlig 9,10,11 | Pierre Kehrli ${ }^{5}$ | François Proust ${ }^{5}$ | Edouard Hirsch ${ }^{4}$ | \\ Izzie-Jacques Namer ${ }^{1,3,12}$
}

\footnotetext{
${ }^{1}$ Department of Biophysics and Nuclear Medicine, University Hospitals of Strasbourg, Strasbourg, France

${ }^{2}$ Department of Pharmacy, University Hospitals of Strasbourg, Strasbourg, France

${ }^{3}$ ICube, University of Strasbourg/CNRS UMR7357, Strasbourg, France

${ }^{4}$ University Hospital of INSERM U 964, Strasbourg, France

${ }^{5}$ Department of Neurosurgery, University Hospitals of Strasbourg, Strasbourg, France

${ }^{6}$ Department of Computer Engineering, Bilkent University, Ankara, Turkey

${ }^{7}$ Computational Biology Department, Carnegie Mellon University, Pittsburgh, PA, USA

${ }^{8}$ Department of Pathology, University Hospitals of Strasbourg, Strasbourg, France

${ }^{9}$ INSERM U1129, Paris, France

${ }^{10}$ Paris Descartes University-Sorbonne Paris Cité, Paris, France

${ }^{11} \mathrm{CEA}$, Gif sur Yvette, France

${ }^{12}$ Federation of Translational Medicine of Strasbourg (FMTS), Faculty of Medicine, University of Strasbourg, Strasbourg, France
}

\section{Correspondence}

Julien Detour, Department of Pharmacy, University Hospitals of Strasbourg, Strasbourg, France. Email: julien.DETOUR@chru-strasbourg. fr

\section{Summary}

Objective: Within a complex systems biology perspective, we wished to assess whether hippocampi with established neuropathological features have distinct metabolome. Apparently normal hippocampi with no signs of sclerosis (noHS), were compared to hippocampal sclerosis (HS) type 1 (HS1) and/or type 2 (HS2). Hippocampus metabolome from patients with epilepsy-associated neuroepithelial tumors (EANTs), namely, gangliogliomas (GGs) and dysembryoplastic neuroepithelial tumors (DNTs), was also compared to noHS epileptiform tissue.

Methods: All patients underwent standardized temporal lobectomy. We applied ${ }^{1} \mathrm{H}$ high-resolution magic angle spinning nuclear magnetic resonance (HRMAS NMR) spectroscopy to 48 resected human hippocampi. NMR spectra allowed quantification of 21 metabolites. Data were analyzed using multivariate analysis based on mutual information.

Results: Clear distinct metabolomic profiles were observed between all studied groups. Sixteen and 18 expected metabolite levels out of 21 were significantly different for HS1 and HS2, respectively, when compared to noHS. Distinct concentration variations for glutamine, glutamate, and $\mathrm{N}$-acetylaspartate (NAA) were observed between HS1 and HS2. Hippocampi from GG and DNT patients showed 7 and 11 significant differences in metabolite concentrations when compared to the same group, respectively. GG and DNT had a clear distinct metabolomic profile, notably regarding choline compounds, glutamine, glutamate, aspartate, and taurine. Lactate and acetate underwent similar variations in both groups.

Significance: HRMAS NMR metabolomic analysis was able to disentangle metabolic profiles between HS, noHS, and epileptic hippocampi associated with EANT. HRMAS NMR metabolomic analysis may contribute to a better identification of abnormal biochemical processes and neuropathogenic combinations underlying mesial temporal lobe epilepsy.

\section{K E Y W O R D S}

hippocampal sclerosis, HRMAS NMR, long-term epilepsy-associated tumor, mesial temporal lobe epilepsy, metabolomics 


\section{1 | INTRODUCTION}

The hippocampus remains the most widely studied brain region in both human and experimental epilepsy. Hippocampal sclerosis (HS) is the most common histopathologic abnormality of drug-resistant epilepsy with temporomesial seizures (mesial temporal lobe epilepsy [mTLE] $)^{1,2}$ In large surgical epilepsy series, HS incidence varies from $33 \%^{3}$ to $61 \% .^{4}$ Epilepsy-associated neuroepithelial tumors (EANTs), especially gangliogliomas (GGs) and dysembryoplastic neuroepithelial tumors (DNTs), are the second most frequent category in epilepsy surgery series. ${ }^{5}$ Their relative incidence in large surgical epilepsy varies from $6 \%$ to $49 \%$ for GG and from $7 \%$ to $80 \%$ for DNT (with mixed form of GG and DNT representing $2 \%$ to $6 \%)^{6}$ Actually, these rare tumors could be considered within a broad histopathological spectrum that is highly epileptogenic, with a predominant location in the temporal lobe $(77 \%$ reported by Blümckle et al. ${ }^{5}$ ), leading clinically to mTLE syndrome. Clinical mTLE may arise from tumoral and nontumoral biochemical processes. Nonetheless the biological behavior of these EANTs is not completely understood.

The neurometabolic hypothesis of epilepsy stems from a variety of evidence including human physiological and biochemical measurements, imaging data, as well as from different animal models. ${ }^{7}$ Major evidences are hypometabolic brain areas as assessed by fluorodeoxyglucose $\left({ }^{18} \mathrm{~F}-\mathrm{FDG}\right)$ using positron emission tomography (PET) and substantial dysfunction at the level of the glial-neuronal unit (GNU) as assessed by abnormal neurotransmission and metabolic cycling. Within this neurometabolic hypothesis, functional imaging such as magnetic resonance spectroscopy (MRS) is recognized as a powerful tool for neurometabolic investigations. These investigations reported abnormalities of brain biochemical processes based on in vivo metabolite identification and quantification. $\mathrm{N}$-acetylaspartate (NAA), creatine, choline-containing compounds, and lactate are the most widely studied metabolites due to their robust detection in vivo and relatively high concentration. An index related to NAA, which is produced in the mitochondria of neurons, has been proposed as an index reflecting neuronal dysfunction in epileptic networks. ${ }^{8}$ Besides in vivo applications, ex vivo nuclear magnetic resonance (NMR) spectroscopy studies have largely contributed to a better understanding of biochemical abnormalities in several epileptic syndromes. ${ }^{9-15}$ These studies used "classical" liquid NMR that requires previous metabolite extraction step from cerebral tissues such as oxidation, separation, and/or lyophilization.

The aim of the present study was the use of ${ }^{1} \mathrm{H}$ highresolution magic angle spinning $\left({ }^{1} \mathrm{H}\right.$ HRMAS) NMR to analyze intact human hippocampi within the syndrome of

\section{Key Points}

- Systems biology approaches for the study of mesial temporal lobe epilepsy (mTLE) remain an emerging field

- HRMAS NMR and multivariate analysis based on metabolic network highlighted metabolomic differences between type 1 and type 2 hippocampal sclerosis

- The same analysis highlighted specific metabolomic pattern differences between ganglioglioma and dysembryoplastic neuroepithelial tumors

- Concentration variations of 4 metabolites, namely, glutamate, glutamine, NAA, and lactate, were able to discriminate all pathological groups

- Metabolomics contribute to identify biochemical disturbances in cerebral samples from patients with mTLE

intractable mTLE recommended for surgery in order to compare the metabolomic profile of EANT (GG and DNT) and nontumoral (HS and apparently normal) epileptiform tissues.

\section{2 | MATERIAL AND METHODS}

\subsection{Patients and hippocampi specimens}

Forty-eight patients with medically intractable mTLE and selected for epilepsy surgery were included in this study. The patients' characteristics are detailed in Table 1. For all patients, the same procedure was planned for surgery and consisted of a standardized "en bloc" amygdalohippocampectomy of variable length extending to the anterior temporal pole. All tissue specimens were collected during surgery right after removal and were snap-frozen in liquid nitrogen for further neuropathological examination and HRMAS NMR analysis. Patients were divided into the following 5 groups according to their medical and imaging records, and their hippocampal neuropathological status:

- The apparently normal epileptiform tissue group (noHS; $\mathrm{n}=10$ ) was composed of mTLE patients with no signs of HS from either neuropathological or imaging data $(\mathrm{n}=10)$. In this group, the hippocampus was resected because of the electroclinical evidence of its involvement in seizure generation.

- HS groups with 2 subgroups according to the International League Against Epilepsy (ILAE) classification $^{16,17}$ : type $1(\mathrm{HS} 1 ; \mathrm{n}=20)$ with severe neuronal cell loss and gliosis within the CA1 and CA4 subregions, 
TA B L E 1 Demographic and clinical features of the study population

\begin{tabular}{|c|c|c|c|c|c|c|c|}
\hline & $\begin{array}{l}\text { noHS } \\
(n=10)\end{array}$ & $\begin{array}{l}\text { HS1 } \\
(n=20)\end{array}$ & $\begin{array}{l}\text { HS2 } \\
(n=6)\end{array}$ & $\begin{array}{l}\text { DNT } \\
(n=5)\end{array}$ & $\begin{array}{l}\text { GG } \\
(n=7)\end{array}$ & $\begin{array}{l}P \text { - } \\
\text { value }\end{array}$ & $\begin{array}{l}P \text {-value } \\
\text { (without the } \\
\text { GG group) }\end{array}$ \\
\hline $\begin{array}{l}\text { Median age at seizure } \\
\text { onset (years } \pm \mathrm{SD})\end{array}$ & $16 \pm 8.45$ & $10 \pm 10.3$ & $4 \pm 8.5$ & $17 \pm 5.6$ & $2 \pm 2.7$ & .008 & n.s. \\
\hline Duration of epilepsy (years $\pm \mathrm{SD}$ ) & $17 \pm 8.4$ & $22 \pm 9.5$ & $31 \pm 19.1$ & $7 \pm 11.4$ & $8 \pm 9.0$ & .012 & n.s. \\
\hline$<10$ years $(\mathrm{n}=14)$ & 3 & 1 & 1 & 4 & 5 & .001 & .003 \\
\hline$>20$ years $(n=24)$ & 3 & 15 & 4 & 1 & 1 & .005 & .016 \\
\hline $\begin{array}{l}\text { Estimated seizures } \\
\text { (frequency/month } \pm \mathrm{SD} \text { ) }\end{array}$ & $10 \pm 24.3$ & $5 \pm 21.9$ & $11 \pm 24.3$ & $11.5 \pm 20.7$ & $5 \pm 9.65$ & n.s. & n.s. \\
\hline$>30$ & 1 & 3 & 1 & 2 & 1 & n.s. & n.s. \\
\hline $\begin{array}{l}\text { Reported febrile convulsion } \\
\text { before } 2 \text { years }\end{array}$ & 2 & 15 & 2 & 0 & 0 & $<.001$ & .003 \\
\hline
\end{tabular}

DNT, dysembryoplastic neuroepithelial tumors; GG, gangliogliomas; HS1, type 1 hippocampal sclerosis; HS2, type 2 hippocampal sclerosis; noHS, absence of hippocampal sclerosis; n.s., not significant.

and type $2(\mathrm{HS} 2 ; \mathrm{n}=6)$ with cell loss and gliosis predominant in CA1.

- EANT group with 2 subgroups according to the 2007 WHO classification ${ }^{18}$ : the GG group $(n=7)$ and the DNT group $(n=5)$. In these cases, the lesions were exclusively localized within mesiotemporal structures, eventually spreading to the temporal lobe.

Details of mTLE patient characteristics within each studied group are shown in Table S1.

\section{2 | Sample preparation and HRMAS NMR data acquisition}

Hippocampal biopsies were introduced into a $30 \mu \mathrm{L}$ Kelf insert (weight $15-20 \mathrm{mg}$ ), and $10 \mu \mathrm{L}$ of buffered $\mathrm{D}_{2} \mathrm{O}$ was added to the insert. The insert ensures that the entire biopsy is detected by the radiofrequency coil of the probe and that no leak occurs during the HRMAS analysis. The inserts containing the biopsy were then stored at $-80^{\circ} \mathrm{C}$. Shortly before the HRMAS analysis, the insert was placed into a $4 \mathrm{~mm} \quad \mathrm{ZrO}_{2}$ rotor. All HRMAS NMR spectra were acquired on a Bruker (Karlsruhe, Germany) Avance III 500 spectrometer (installed at Hautepierre Hospital, Strasbourg University Hospitals) operating at a proton frequency of $500.13 \mathrm{MHz}$ and equipped with a 4-mm triple resonance gradient HRMAS probe $\left({ }^{1} \mathrm{H},{ }^{13} \mathrm{C}\right.$, and $\left.{ }^{31} \mathrm{P}\right)$. The temperature was maintained at $4{ }^{\circ} \mathrm{C}$ throughout the acquisition time to reduce the effects of tissue degradation during spectrum acquisition. All NMR experiments were conducted on samples spinning at $3502 \mathrm{~Hz}$ to keep the rotation sidebands out of the spectral region of interest. For each biopsy sample, a 1-dimensional (1D) proton spectrum was acquired using a Carr-Purcell-Meiboom-Gill (CPMG) sequence. The inter-pulse delay between the $180^{\circ}$ pulses of the CPMG pulse train was synchronized with the sample and set to $285 \mu$ s to eliminate signal losses due to B1 inhomogeneities. The number of loops was set to 328 , giving the CPMG pulse train a total length of $93 \mathrm{msec}$. Parameters for the CPMG experiment were the following: sweep width $14.2 \mathrm{ppm}$, number of points $32 \mathrm{k}$, relaxation delay $2 \mathrm{~s}$, and acquisition time $2.3 \mathrm{~s}$. A total of 128 free induction decay (FID) were acquired resulting in an acquisition time of 10 minutes. All spectra were recorded in such a manner that only a zero-phase order correction was necessary to properly phase the spectrum. The FID was multiplied by an exponential weighing function corresponding to a line broadening of $0.3 \mathrm{~Hz}$ prior to Fourier transformation. Spectra were referenced by setting the lactate doublet chemical shift to $1.33 \mathrm{ppm}$. To confirm resonance assignments in a few representative samples, 2-dimensional (2D) heteronuclear experiments $\left({ }^{1} \mathrm{H}-{ }^{13} \mathrm{C}\right)$ were also recorded immediately after ending the 1D acquisition. More details can be found in a previous article from our group. ${ }^{19}$ Metabolites were assigned using standard metabolite chemical shift tables available in the literature. ${ }^{19,20}$ 


\section{3 | HRMAS NMR data processing and statistical analyses}

The metabolites were quantified using the pulse length-based concentration determination (PULCON) method, a very accurate quantification method. ${ }^{21}$ The metabolites were quantified using an external reference standard of lactate $(3 \mu \mathrm{mol})$ scanned under the same analytical conditions as the tissue samples. Quantification was performed for each metabolite on ${ }^{1} \mathrm{H}$ groups listed in Table S2. The spectra were normalized according to sample weight. Peaks of interest were automatically defined using an in-house program under MATLAB 7.0 (MathWorks, Natick, MA, USA). The peak integration for each metabolite was then compared to the peak integration of the lactate reference and corrected according to the number of protons. The results are expressed in nmol. $\mathrm{mg}^{-1}$ of tissue.

The Algorithm to Determine Expected Metabolite Level Alterations (ADEMA) based on mutual information was applied to the metabolite quantification values. ${ }^{22}$ ADEMA includes information on the metabolic pathway in a unidirectional or bidirectional manner. The network was constructed using the Kyoto Encyclopedia of Genes. ${ }^{23,24}$ Using the metabolic network topology, the ADEMA algorithm evaluates the change in groups of metabolites between concentration data from 2 experimental groups instead of analyzing metabolite concentrations one by one. Based on mutual information, the Algorithm determines whether some metabolites are biomarkers when considered together, and it can predict the direction of the expected change per metabolite depending on the metabolic network topology considered. The metabolic network considered herein is described in Figure S1. Various groups of metabolites related to different metabolic pathways were compared:

- Aspartate, taurine

- Aspartate, arginine, acetate, NAA

- Glucose, glycine

- Glucose, valine

- Glucose, acetate

- Glucose, lactate

- Valine, lactate, alanine

- Glucose, myoinositol, ascorbate, glutathione, glycine, glutamate

- $\gamma$-Aminobutyric acid (GABA), glutamate, glutamine

- Glutamine, glutamate, lactate

- Glutamate, glutamine, glycine

- Glutamate, arginine, glycine, creatine

- Choline, phosphocholine, glycerophosphocholine, total choline

Statistical analyses performed on data from the study population were conducted under SPSS 17 (SPSS Inc., Chicago, IL, USA). Kruskal-Wallis analysis of variance was used for nonparametric measures. Categorical variables were analyzed by means of the Pearson's chi-square. The level of significance was set at 0.05 .

\section{3 | RESULTS}

All spectra obtained from the 48 patients' hippocampi were of standard quality according to water signal suppression. The representative 1D HRMAS NMR spectra from each experimental group are shown in Figure 1. A total of 21 metabolites were quantified within the range of 0.5 to $4.5 \mathrm{ppm}$ (Table 2). Other metabolites were identified, but due to partial overlap and/or poor resolution leading to quantification problems, these metabolites were not integrated into any further analysis. Table 1 (right column) indicated that median age at seizure onset, age at surgery, duration of epilepsy, and estimated numbers of seizures per month were not significantly different across noHS, HS1, HS2, and DNT groups. Only sample sizes of patients with less than 10 years and more than 20 years of epilepsy duration were significantly different across groups. When including the GG group in the statistical analysis, almost all items showed statistically significant differences except gender, lesion lateralization, and estimated number of seizures per month.

\section{1 mTLE with and without HS}

The upper part of Table 3 summarizes the statistical results according to the ADEMA network analysis obtained on metabolite concentration comparisons from non-tumor-related mTLE patients (noHS, HS1, and HS2 groups). The HS (HS1 + HS2) group, compared to the noHS group, was characterized by an elevated concentration for glutamine, glutamate, and glutathione and a low concentration for acetate, alanine, arginine, ascorbate, glycine, NAA, phosphocholine, taurine, total choline, and valine. Glutamine was higher in the HS1 group, and glutamate was higher in the HS2 group. NAA was lower in the HS1 group. In all contrasts presented, aspartate never showed any statistical variations between the 2 groups for any of the statistical analyses. An ADEMA analysis was also conducted according to seizure frequency across groups: high (more than 30 seizures per month) vs low (less than 5 seizures per month). For all groups (noHS, HS1, and HS2) high seizure frequency was associated with elevated concentrations of acetate, alanine, creatine, glutamine, glycerophosphocholine, taurine, and valine. In the HS group (HS1 + HS2), high seizure frequency was associated with a low level of NAA and aspartate.

Regarding epilepsy duration, for all studied groups, high duration $(>20$ years) compared to low duration $(<10$ years) was associated with high concentrations of glucose, lactate, and taurine and low concentrations of aspartate and NAA. 
F I G URE 1 Example of ${ }^{1} \mathrm{H}$ NMR HRMAS spectra of hippocampus from mTLE patients. A, Hippocampus with no sign of sclerosis (noHS); B, type 1 hippocampal sclerosis (HS1); C, type 2 hippocampal sclerosis (HS2); D, dysembryoplastic neuroepithelial tumors (DNTs); E, ganglioglioma (GG). (1) Acetate; (2) alanine; (3) arginine; (4) ascorbate; (5) aspartate; (6) choline; (7) creatine; (8) GABA; (9) $\beta$-glucose; (10) glutamate; (11) glutamine; (12) glutathione; (13) glycerophosphocholine; (14) glycine;

(15) lactate; (16) myoinositol; (17) NAA;

(18) phosphocholine; (19) taurine; (20)

valine; and (21) choline-containing compounds (tCho)
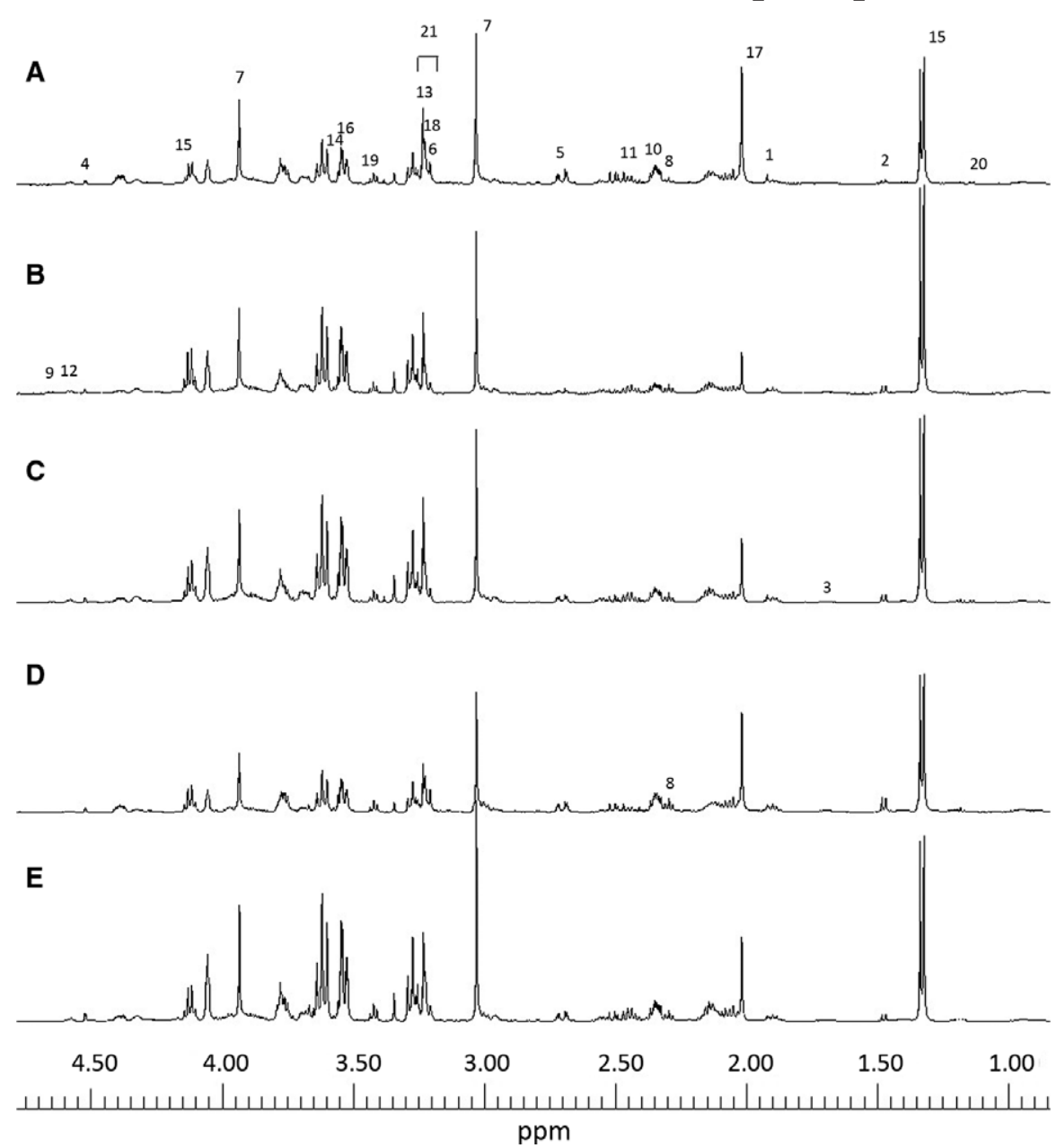

\section{2 | mTLE with EANT}

The lower part of Table 3 summarizes statistical results according to the ADEMA network analysis obtained on DNT and GG. Compared to noHS tissue, DNTs were characterized by high concentrations of alanine, arginine, ascorbate, and lactate and low concentrations of acetate, glutamine, and glutathione. Compared to noHS tissue, GGs were characterized by high concentrations of glutamine, glutathione, and lactate and low concentrations of aspartate, glutamate, glycine, myoinositol, taurine, and phosphocholine. Altogether, there were fewer significant variations of metabolites concentrations between hippocampi from EANTs compared to noHS than HS (HS1\&Hs2) compared to the same group (noHS), especially considering the low level.

\section{4 | DISCUSSION}

As far as we know, no previous study applied ${ }^{1} \mathrm{H}$ NMR HMRAS analysis to intact resected human hippocampi from drug-resistant mTLE. Moreover, no previous data from high-resolution ex vivo NMR studies applied multivariate analysis on such brain samples according to their sclerotic status. We report here direct comparisons of the NMR metabolomic profile of "epileptic" hippocampi from mTLE patients related or not to tumoral processes.

HRMAS NMR spectroscopy presents the advantages of fast preparation and rapid biochemical characterization of intact tissue (less than 20 minutes) in optimal conditions $\left(-20^{\circ} \mathrm{C}\right.$ for tissue preparation, $4^{\circ} \mathrm{C}$ for NMR acquisition). This method is now widely used, as reported in the literature, most particularly in cancer research, opening the perspective of real-time metabolic profiling during surgery, called metabolomics-guided surgery. ${ }^{25}$ The repetition time used for this study $(2 \mathrm{~s})$ and the total acquisition time (10 minutes) are hence a good compromise when quantifying the metabolites using HRMAS NMR spectroscopy of intact tissue. However, in these conditions, we cannot see the total metabolite pool but only the most mobile part of it, that is, the soluble part of metabolites, as opposed to bound metabolites to proteins. Furthermore, these are underestimated by approximately $20 \%$ compared with data from studies performing tissue extractions (data not shown). 
TA B LE 2 Metabolite concentrations in intact human hippocampi as measured by ${ }^{1} \mathrm{H}$ HRMAS NMR

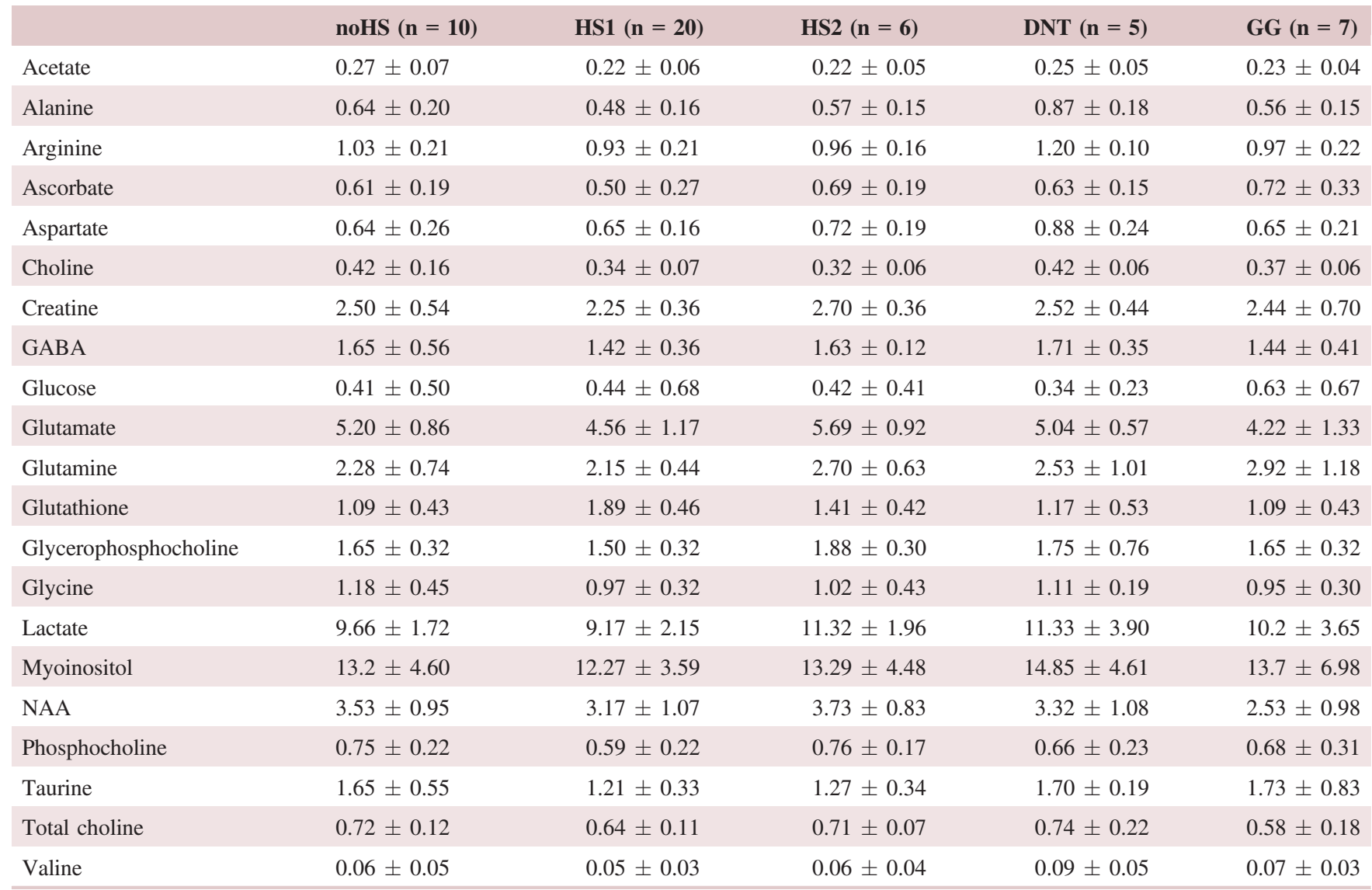

Values are given in $\mathrm{nmol} \cdot \mathrm{mg}^{-1} \pm \mathrm{SD}$.

DNT, dysembryoplastic neuroepithelial tumor; GG, ganglioglioma; HS1, type 1 hippocampal sclerosis; HS2, type 2 hippocampal sclerosis; noHS, absence of hippocampal sclerosis.

The major limitation could be the known confounding effect of antiepileptic drugs on neurometabolism, since patients were under pharmacological treatment at the time of surgery. However, all patients had refractory epilepsy not controlled at the date of surgery. Moreover, because the patients were anesthetized during the surgical procedure, these drugs may also have affected the metabolite level within resected brain tissue samples. To limit artifacts, the same procedure was applied to all patients. We only used one hippocampal sample for each patient, namely CA1 based on routine surgical practices. Analysis of other hippocampal subfields would have been interesting to study, since neuronal loss, gliosis, and metabolite concentration may vary according to the anteroposterior axis of the hippocampus. ${ }^{6,26,27}$ However, tissue harvest was sometimes limited due to diagnosis need for clinical patient management. Neuropathological examinations and longterm biopsy storage already require high quality cerebral sample. Despite the relatively homogenous MTLE clinical syndrome, ${ }^{1} \mathrm{H}$ HRMAS NMR metabolomics was able to disentangle the metabolic profile between HS, nonsclerotic hippocampus (described previously as cryptogenic), and hippocampus associated with EANTs.
Reported metabolite concentrations in human hippocampi are globally in agreement with the few available comparable liquid NMR studies. The concentrations reported for alanine, NAA, glutamate, taurine, and lactate exhibited values within the range of the study by Peeling and Sutherland. ${ }^{12}$ Only creatine in the present study was lower compared to the study of Peeling and Sutherland. Aspartate, acetate, NAA, and glutamine concentrations were lower in the present study than the levels reported by Petroff et al. ${ }^{9-11}$ and Vielhaber et al., ${ }^{15}$ but alanine, glutamate, lactate, and taurine were within the same range. The level of NAA was within the range reported by Petroff et al., ${ }^{28}$ who already indicated that ex vivo NMR quantification of this metabolite in the hippocampi of mTLE patients was below the values reported in the same brain region of control subjects using in vivo MR spectroscopy. ${ }^{27,29}$ Therefore, variations in metabolite concentration across studies can be interpreted according to several methodological considerations. It should be noted that all previous studies performed analyses in a liquid NMR spectrometer; none used entirely frozen samples as can be done using HRMAS NMR. None analyzed as many brain biopsy samples as in the present study. Moreover, the 


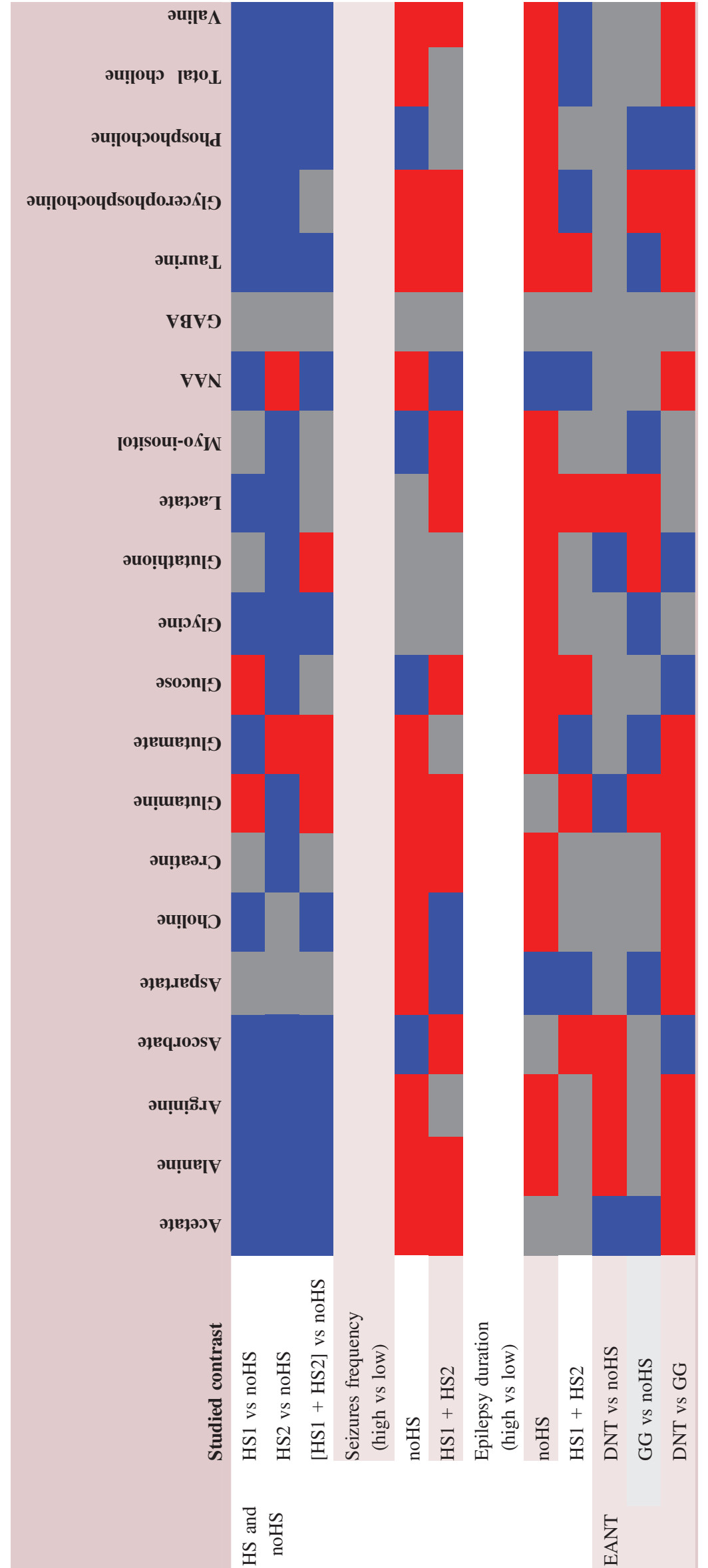

ठํ छ

क ग

$\exists$.

a.

产

चै

点

王

道节

.

을 듕

跣

ठ․

戈

次

들

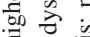

安完递

可

을 뜰

을 흠

可

擘

\%

융

言 。

逑

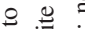

on

过

政

ชै 8 है

ㅇํㅇ

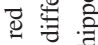

tᄒ

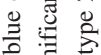

$\exists \frac{50}{5}$ i

요

递

흔

零

응 댄

言

年

$\$$

政

㱐

竞

B.

楶焉

ज्ञ

言言总

䒭 总 
reproducibility of the hippocampal subfield analyzed is difficult to appreciate across studies, and management of resected tissue from surgery is sometimes difficult to standardize and may lead to relative variability across studies. ${ }^{16}$

\subsection{Metabolomic comparison between hippocampi with and without HS}

Two-by-two statistical comparisons of metabolite concentrations within hippocampi from mTLE patients according to their sclerotic status exhibited limited significant differences. The ADEMA network analysis highlights many more differences between groups. Type 1 and type 2 sclerotic hippocampi from mTLE patients exhibited lower concentrations for 14 and 16 metabolites, respectively, out of 21 when compared to hippocampi with no signs of sclerosis. The most discriminant metabolites among the 3 groups were glutamine, glutamate, and NAA. Glutamate and NAA were lower in the HS1 group when compared to noHS, but this was not the case in the HS2 group. The reverse pattern was observed for glutamine. Moreover, several related metabolic pathways exhibited higher concentrations for key metabolites in the noHS group compared to the HS group (regardless of their type) within the phospholipid metabolism pathway (glycerophosphocholine, phosphocholine, and total choline) and specific amino acid metabolism (ascorbate, taurine, and glutathione). Such results could be partially expected, since HS is associated with severe cell loss (mostly neurons) as well as with axonal sprouting and granule cell dispersion. ${ }^{2}$ Hence, diminished global "density" of cellular metabolism leading to decreased metabolite concentrations could have been expected in HS. This view may be counterbalanced by gliosis within the CA1 subfield of HS1, which is associated with dense fibrillary, reactive glial cells and, as reported more recently, with inflammatory metabolism processes. ${ }^{2,30}$ However, previous ex vivo NMR studies of human hippocampi from mTLE patients reported only a few significant concentration changes. ${ }^{14}$ Such results might be correlated with the statistical method applied.

The high frequency of epileptic seizures was associated with increased concentrations for acetate, alanine, creatine, glutamine, taurine, glycerophosphocholine, and valine in HS and noHS hippocampi. We also observed low levels of NAA and aspartate and high levels of lactate, myoinositol, and ascorbate in HS. These results are difficult to interpret due to a lack of comparable data in the literature and the subjective clinical quantification of seizure frequency based on medical records. A similar contrast was explored regarding epilepsy duration: more than 20 years vs less than 10 years since epilepsy onset. Lower concentrations for aspartate and NAA were associated with long epilepsy duration in the HS and noHS groups.

\subsection{Metabolomics of EANT hippocampi}

Regarding DNT hippocampi, the present study has partially confirmed the in vivo MR spectroscopy results reported by Bulakbasi et al., ${ }^{31}$ who found normal NAA-related ratios but an increase of the myoinositol/creatine ratio. Tzika et al. ${ }^{32}$ found multiple up-regulated genes in anaplastic ganglioglioma brain tumors vs epileptic tissues related to primary metabolism and cellular metabolism, with significantly increased concentrations of lactate, alanine, glutamate, choline, and phosphocholine and decreased concentrations of NAA and creatine. They suggest a rapid phospholipid turnover that coincides with up-regulated cell proliferation. The present study confirms these observations. Moreover, our study highlights a clear difference between DNT and GG regarding choline metabolism. No choline-containing compounds had concentration variations within the DNT group according to the reported contrast. We should also mention that some clinical features showed significant differences between both groups (median age at onset, at surgery, and epilepsy duration) due to distinct surgical management between DNTs and GGs. These differences may also contribute to reported metabolomic patterns.

It should be noted that in both patients with GGs or DNTs, glycine did not undergo any significant concentration variations compared to hippocampi with no sign of sclerosis. Glycine has been reported as a potential biomarker in low-grade gliomas and brain metastases. ${ }^{33}$ Because mixed DNT and GG have been reported ${ }^{34,35}$ and are considered as contentious entities, NMR HRMAS might contribute to identify specific metabolic disturbance between the simple and complex form of DNT. To this extent, merging ex vivo and in vivo NMR sources, as well as neuropathological and/or genomics data may contribute to improving EANT diagnosis. ${ }^{32,36,37}$ For example, relatively good correlations have been found between in vivo MRS and ex vivo HRMAS results in adult human glioma. ${ }^{38}$ Because rare patients with DNTs have been reported to express aggressive behavior, further work will be necessary with patient follow-up or outcomes to assess the medical value of HRMAS NMR for EANT patient management.

Altogether, using hippocampi from mTLE patients with no sign of sclerosis as "reference group," HRMAS NMR showed a specific metabolomic pattern for each studied group, namely type 1 and type $2 \mathrm{HS}$, DNT, and GG. Our metabolomic network analysis based on mutual information strikingly identified an unequivocal metabolic pattern among groups based on only 4 metabolites, namely, glutamine, glutamate, NAA, and lactate. Hence Table 4 summarizes the critical role of the concentration change in these 4 metabolites in each epilepsy syndrome studied as a 
TA B LE 4 Summary of the metabolic variations found in 4 metabolites for each studied group compared to hippocampi with no sign of sclerosis

$\begin{array}{llll} & \text { Glutamine } & \text { Glutamate } \\ \mathrm{HS} 1 & \text { NAA } \\ \mathrm{HNT} 2 & & 3 & =\end{array}$

DNT, dysembryoplastic neuroepithelial tumor; GG, ganglioglioma; HS1, type 1 hippocampal sclerosis; HS2, type 2 hippocampal sclerosis.

clear and simple take-home message. The glutamateglutamine cycle in glial cells is affected in epileptic tissue. ${ }^{10}$ NAA is considered as a neuronal marker and is a direct precursor of the enzymatic synthesis of the most concentrated neuropeptide in the human brain. Finally, lactate is often involved in energy neurometabolism via the astrocyte-neuron lactate shuttle and in oncological metabolism via the Warburg effect. ${ }^{39,40}$

\section{5 | CONCLUSION}

This study is an exploratory metabolomic analysis of intact epileptic hippocampi of patients with drug-resistant mTLE aimed at comparing samples with and without EANT, and with and without HS. ${ }^{1} \mathrm{H}$ HRMAS NMR was able to disentangle metabolic profiles between type 1 and type 2 sclerotic hippocampus. This study also provides relevant metabolic information to discriminate hippocampi from patients with GG and DNT. Hence ex vivo HMRAS NMR may contribute to EANT classification, especially with respect to the most complex form. This approach could contribute to an advanced and innovative research strategy for epilepsy, taking into account all systems biology. HS is based on imaging data and neuropathological criteria primarily recommended by the ILAE, which has attempted to overcome diagnosis difficulties on brain samples. HRMAS NMR metabolomics may contribute to a better identification of pathogenic combinations underlying HS and therefore its classification. Nonetheless, further work with larger cohorts is needed together with a consensus regarding methodological and statistical issues.

\section{ETHICS APPROVAL AND CONSENT TO PARTICIPATE}

The Ethics Committee of Strasbourg (Comité de Protection des Personnes "Est IV") approved the study ( ${ }^{\circ}$ 09/39, 13.10.2009). The declaration at the ministerial level and the authorization from the Tumor Bio-Bank (Centre de
Ressources Biologiques) of the University Hospitals of Strasbourg correspond to the following number: AC 2008438/DC 2009-1016. Written informed consent was obtained from all the patients included. For this investigation, the tissue samples were obtained from the Tumor Bio-bank (Centre de Ressources Biologiques) of the University Hospitals of Strasbourg.

\section{ACKNOWLEDGMENTS}

We gratefully acknowledge F.M. Moussallieh and E. Ruhland for skillful technical assistance and K. Elbayed for the Matlab scripts that he developed to quantify the metabolites. The laboratory staff of the Tumor Bio-bank (Centre de Ressources Biologiques) of the University Hospitals of Strasbourg are also gratefully acknowledged for their technical assistance.

\section{DISCLOSURE}

None of the authors has any conflicts of interest to disclose. We confirm that we have read the Journal's position on issues involved in ethical publication, and affirm that this report is consistent with those guidelines.

\section{REFERENCES}

1. Wieser H-G and ILAE Commission on Neurosurgery of Epilepsy. ILAE Commission Report. Mesial temporal lobe epilepsy with hippocampal sclerosis. Epilepsia. 2004;45:695-714.

2. Thom M. Review: hippocampal sclerosis in epilepsy: a neuropathology review. Neuropathol Appl Neurobiol. 2014;40: 520-43.

3. Blümcke I, Coras R, Miyata H, et al. Defining clinico-neuropathological subtypes of mesial temporal lobe epilepsy with hippocampal sclerosis. Brain Pathol. 2012;22:402-11.

4. de Tisi J, Bell GS, Peacock JL, et al. The long-term outcome of adult epilepsy surgery, patterns of seizure remission, and relapse: a cohort study. Lancet. 2011;378:1388-95.

5. Blümcke I, Aronica E, Urbach H, el al.A neuropathology-based approach to epilepsy surgery in brain tumors and proposal for a new terminology use for long-term epilepsy-associated brain tumors. Acta Neuropathol. 2014;128:39-54.

6. Thom M, Blümcke I, Aronica E. Long-term epilepsy-associated tumors. Brain Pathol. 2012;22:350-79.

7. Pan JW, Williamson A, Cavus I, et al. Neurometabolism in human epilepsy. Epilepsia. 2008;49(Suppl 3):31-41.

8. Pan JW, Kuzniecky RI. Utility of magnetic resonance spectroscopic imaging for human epilepsy. Quant Imaging Med Surg. 2015;5:313-22.

9. Petroff OAC, Spencer DD, Alger JR, et al. High-field proton magnetic resonance spectroscopy of human cerebrum obtained during surgery for epilepsy. Neurology. 1989;39:1197-202.

10. Petroff OAC, Errante LD, Rothman DL, et al. Glutamate-glutamine cycling in the epileptic human hippocampus. Epilepsia. 2002;43:703-10. 
11. Petroff OAC, Errante LD, Kim JH, et al. N-acetyl-aspartate, total creatine, and myo-inositol in the epileptogenic human hippocampus. Neurology. 2003;60:1646-51.

12. Peeling J, Sutherland G. ${ }^{1} \mathrm{H}$ magnetic resonance spectroscopy of extracts of human epileptic neocortex and hippocampus. Neurology. 1993;43:589-94.

13. Maxwell RJ, Martínez-Pérez I, Cerdán S, et al. Pattern recognition analysis of ${ }^{1} \mathrm{H}$ NMR spectra from perchloric acid extracts of human brain tumor biopsies. Magn Reson Med. 1998;39:869-77.

14. Aasly J, Silfvenius H, Aas TC, et al. Proton magnetic resonance spectroscopy of brain biopsies from patients with intractable epilepsy. Epilepsy Res. 1999;35:211-7.

15. Vielhaber S, Niessen HG, Debska-Vielhaber G, et al. Subfieldspecific loss of hippocampal $\mathrm{N}$-acetyl aspartate in temporal lobe epilepsy. Epilepsia. 2008;2008:40-50.

16. Blümcke I, Aronica E, Miyata H, et al. International recommendation for a comprehensive neuropathologic workup of epilepsy surgery brain tissue: a consensus task force report from the ILAE commission on diagnostic methods. Epilepsia. 2016;57:348-58.

17. Scheffer IE, Berkovic S, Capovilla G, et al. ILAE classification of the epilepsies: position paper of the ILAE commission for classification and terminology. Epilepsia. 2017;58:512-21.

18. Louis DN, Ohgaki H, Wiestler OD, et al. The 2007 WHO classification of tumors of the central nervous system. Acta Neuropathol. 2007;114:97-109.

19. Detour J, Elbayed K, Piotto M, et al. Ultrafast in vivo microwave irradiation for enhanced metabolomic stability of brain biopsy samples during HRMAS NMR analysis. J Neurosci Methods. 2011;201:89-97.

20. Wishart DS, Jewison T, Guo AC, et al. HMDB 3.0-the human metabolome database in 2013. Nucleic Acids Res. 2013;41: D801-7.

21. Dreier L, Wider G. Concentration measurements by PULCON using X-filtered or 2D NMR spectra. Magn Reson Chem. 2006;44:S206-12.

22. Cicek AE, Bederman I, Henderson L, et al. ADEMA: an algorithm to determine expected metabolite level alterations using mutual information. PLoS Comput Biol. 2013;9:e1002859.

23. Kanehisa M, Goto S, Sato Y, et al. Data, information, knowledge and principle: back to metabolism in KEGG. Nucleic Acids Res. 2014;42:D199-205.

24. Selway ZZ. Metabolism at a glance, 3rd edn. Malden (MI): Blackwell Publishing; 2014.

25. Battini B, Faitot F, Imperiale A, et al. Metabolomics approach in pancreatic adenocarcinoma: tumor metabolism predicts clinical outcome of patients. BMC Med. 2017;15:56.

26. Starck G, Vkhoff-Baaz B, Ljungberg M, et al. Anterior to posterior hippocampal MRS metabolite difference is mainly a partial volume effect. Acta Radiol. 2010;51:351-9.

27. Mueller SG, Ebel A, Barakos J, et al. Widespread extrahippocampal NAA/(Cr+Cho) abnormalities in TLE with and without mesial temporal sclerosis. J Neurol. 2011;258:603-12.

28. Petroff OAC, Errante LD, Rothman DL, et al. Neuronal and glial metabolite content of the epileptogenic human hippocampus. Ann Neurol. 2002;52:635-42.

29. Namer IJ, Bolo NR, Sellal F, et al. Combined measurements of hippocampal $\mathrm{N}$-acetyl-aspartate and $\mathrm{T}_{2}$ relaxation time in the evaluation of mesial temporal lobe epilepsy. Correlation with clinical severity and memory performance. Epilepsia. 1999;40:1424-32.

30. Yang T, Zhou D, Stefan H. Why mesial temporal lobe epilepsy with hippocampal sclerosis is progressive: uncontrolled inflammation drives disease progression? J Neurol Sci. 2010;296:1-6.

31. Bulakbasi N, Kocaoglu M, Sanal TH, et al. Dysembryoplastic neuroepithelial tumors: proton MR spectroscopy, diffusion and perfusion characteristics. Neuroradiology. 2007;2007:805-12.

32. Tzika AA, Astrakas L, Cao H, et al. Combination of high-resolution magic angle spinning proton magnetic resonance spectroscopy and microscale genomics to type brain tumor biopsies. Int J Mol Med. 2007;20:199-208.

33. Righi V, Andronesi OC, Mintzopoulos D, et al. High-resolution magic angle spinning magnetic resonance spectroscopy detects glycine as a biomarker in brain tumors. Int $\mathrm{J}$ Oncol. 2010;36:301-6.

34. Thom M, Toma A, An S, et al. One hundred and one dysembryoplastic neuroepithelial tumors: an adult epilepsy series with immunohistochemical, molecular genetic, and clinical correlations and a review of the literature. J Neuropathol Exp Neurol. 2011;70:859-78.

35. Hirose T, Scheithauer BW. Mixed dysembryoplastic neuroepithelial tumor and ganglioglioma. Acta Neuropathol. 1998;95:649-54.

36. Croitor-Sava A, Martinez-Bisbal MC, Van Huffel S, et al. Ex vivo high resolution magic angle spinning metabolic profiles describe intratumoral histopathological tissue properties in adult human gliomas. Magn Reson Med. 2011;65:320-8.

37. Astrakas L, Blekas KD, Constantinou C, et al. Combining magnetic resonance spectroscopy and molecular genomics offers better accuracy in brain tumor typing and prediction of survival than either methodology alone. Int J Oncol. 2011;38:1113-27.

38. Opstad KS, Wright AJ, Bell BA, et al. Correlations between in vivo (1)H MRS and ex vivo (1)H HRMAS metabolite measurements in adult human gliomas. J Magn Reson Imaging. 2010;31:289-97.

39. Bélanger M, Allaman I, Magistretti PJ. Brain energy metabolism: focus on astrocyte-neuron metabolic cooperation. Cell Metab. 2011;14:724-38.

40. San-Millán I, Brooks GA. Reexamining cancer metabolism: lactate production for carcinogenesis could be the purpose and explanation of the Warburg Effect. Carcinogenesis. 2017;38: 119-33.

\section{SUPPORTING INFORMATION}

Additional Supporting Information may be found online in the supporting information tab for this article.

How to cite this article: Detour J, Bund C, Behr C, et al. Metabolomic characterization of human hippocampus from drug-resistant epilepsy with mesial temporal seizure. Epilepsia. 2018;59:607-616. https://doi.org/10.1111/epi.14000 